\title{
Global dietary calcium intake among adults: a systematic review
}

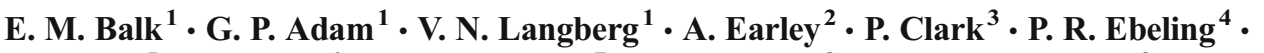 \\ A. Mithal ${ }^{5}$ - R. Rizzoli ${ }^{6}$ - C. A. F. Zerbini ${ }^{7}$ - D. D. Pierroz ${ }^{8}$ - B. Dawson-Hughes ${ }^{9} \cdot$ for the \\ International Osteoporosis Foundation Calcium Steering Committee
}

Received: 26 July 2017 / Accepted: 17 September 2017 /Published online: 12 October 2017

(C) The Author(s) 2017. This article is an open access publication

\begin{abstract}
Low calcium intake may adversely affect bone health in adults. Recognizing the presence of low calcium intake is necessary to develop national strategies to optimize intake. To highlight regions where calcium intake should be improved, we systematically searched for the most representative national dietary calcium intake data in adults from the general population in all countries. We searched 13 electronic databases and requested data from domain experts. Studies were double-screened for eligibility. Data were extracted into a standard form. We developed an interactive global map, categorizing countries based on average calcium
\end{abstract}

Electronic supplementary material The online version of this article (https://doi.org/10.1007/s00198-017-4230-x) contains supplementary material, which is available to authorized users.

E. M. Balk

ethan_balk@brown.edu

1 Center for Evidence Synthesis in Health, Brown University School of Public Health, Providence, RI, USA

2 Evidera, 500 Totten Pond Rd, Waltham, MA, USA

3 Hospital Infantil de México Federico Gómez, Mexico City, Mexico

4 School of Clinical Sciences, Monash University, Clayton, Australia

5 Medanta Medicity, Sector 38, Gurgaon, India

6 Division of Bone Diseases, Geneva University Hospitals and Faculty of Medicine, Geneva, Switzerland

7 Centro Paulista de Investigação Clínica, Sao Paulo, Brazil

8 International Osteoporosis Foundation, Nyon, Switzerland

9 Jean Mayer USDA Human Nutrition Research Center on Aging, Tufts University, Boston, MA, USA intake and summarized differences in intake based on sex, age, and socioeconomic status. Searches yielded 9780 abstracts. Across the 74 countries with data, average national dietary calcium intake ranges from 175 to $1233 \mathrm{mg} /$ day. Many countries in Asia have average dietary calcium intake less than $500 \mathrm{mg} /$ day. Countries in Africa and South America mostly have low calcium intake between about 400 and $700 \mathrm{mg} /$ day. Only Northern European countries have national calcium intake greater than $1000 \mathrm{mg} /$ day. Survey data for three quarters of available countries were not nationally representative. Average calcium intake is generally lower in women than men, but there are no clear patterns across countries regarding relative calcium intake by age, sex, or socioeconomic status. The global calcium map reveals that many countries have low average calcium intake. But recent, nationally representative data are mostly lacking. This review draws attention to regions where measures to increase calcium intake are likely to have skeletal benefits.

Keywords Bone health $\cdot$ Dietary calcium intake $\cdot$ Dietary surveys $\cdot$ Osteoporosis

\section{Introduction}

Calcium intake is one of the many factors affecting the development of peak bone mass and preservation of bone mass in adults. Calcium is an important component of bone, accounting for about 30 to $35 \%$ of its mass and much of its strength. The contribution of dietary calcium intake to bone mass is easiest to document during periods when bone mass is changing rapidly, that is, during adolescence when the skeleton 
gains up to $409 \mathrm{~g}$ per year in boys and $325 \mathrm{~g}$ per year in girls [1], and late in life when bone loss occurs at a rate of about $1 \%$ per year, resulting in calcium loss of approximately $15 \mathrm{~g}$ per year [2]. Low calcium intake in some populations may be adversely affecting the development of peak bone mass in adolescents and young adults and the retention of bone mass in older adults. Recognizing the presence of low calcium intake is a necessary first step in developing culturally appropriate strategies and policies to address the deficiency.

The International Osteoporosis Foundation (IOF) took the initiative to describe dietary calcium intake in the general adult population in different countries based on a systematic review and to present the data on a global map. This study follows on a similar review and map of global vitamin D status conducted in 2011 (https:/www.iofbonehealth.org/facts-and-statistics/ vitamin-d-studies-map) [3]. The aim of the current review was to find the most representative data for each country, regarding average dietary intake of calcium in general population adults. The available studies were used to populate a color-coded global map of average dietary calcium intake per country.

\section{Methods}

We used a systematic review approach to search for, select, and analyze available studies, following, to the extent possible, Institute of Medicine guidance [4]. The protocol was discussed and agreed upon with the Calcium Map Steering Committee of the IOF. The research team (EMB, GPA, VNL, AE) independently conducted the review. Preliminary findings were presented at the World Congress on Osteoporosis, Osteoarthritis, and Musculoskeletal Diseases on March 24, 2017. Attendees were encouraged to inform the team of any missing or erroneous studies.

\section{Data sources}

We searched 13 electronic databases that focus on medical, nutrition, allied health, and global health literature. These included PubMed, Embase, CINAHL, CAB Abstracts, Global Health, Academic Search Premier, Africa-Wide Information, American Bibliography of Slavic and East European Studies, Anthropology Plus, Bibliography of Asian Studies, Environment Index, Humanities \& Social Sciences Index Retrospective, SocINDEX, and LILACS. The searches were conducted on December 16, 2016, and included terms for calcium, micronutrients, dairy, and other calcium-rich foodstuffs; nutrition, health, diet, and food surveys, food frequency questionnaires, and food records; and lists of 154 countries and 158 known national or regional food surveys (Electronic supplementary material-Appendix A). In databases that allowed it, searches were restricted to primary studies or systematic reviews of humans and adults. From relevant reviews and selected primary studies, we searched for additional studies in reference lists. We also conducted web-based searches for national dietary surveys in general and specific named surveys where we had basic information. We also requested known studies from domain experts in the IOF. One author was contacted to help obtain data alluded to in her publication [5]. Data for India and Gambia were obtained for us by an IOF member from the survey researchers directly (Varghese JS et al., Daily adult calcium intake in Indian states based on the NSSO (2012) data, personal communication; Ward K. Gambia. Medical Research Council Lifecourse Epidemiology, University of Southampton, UK. Funded by the Nutrition and Bone Health Research Programme, Medical Research Council (MRC) Elsie Widdowson Laboratory, Cambridge, UK, personal communication).

\section{Study selection}

Four researchers screened abstracts and full-text articles in duplicate. Screening was conducted in two phases. The first phase aimed to find all potentially relevant articles. The second phase selected the one or two most representative studies for each country. To select studies, we formulated an ideal set of eligibility criteria and included the studies that best matched these criteria. For countries that did not have ideal data, we included less representative studies. The ideal eligibility criteria were national (or regional) surveys (designed to be nationally representative) of general population adults $(\geq$ 18 years old) that reported average dietary calcium intake (excluding supplement intake) in milligrams per day or equivalent, surveyed since 2010. Ideally, studies also reported subgroup analyses based on sex, age, and socioeconomic status. For countries that lacked such studies, we allowed nonnational (e.g., city-level) studies and studies of selective populations (e.g., by age or sex). In addition, when necessary, we allowed studies that included children or calcium supplement intake. When studies were not nationally representative, we allowed multiple studies from a single country if the studies were complementary (e.g., represented different regions or age ranges).

We excluded studies that were restricted to children, institutionalized adults or those with comorbidities (e.g., studies of nursing home residents or those with osteoporosis or diabetes), lactating or pregnant women, or if study participants had to undergo imaging testing (e.g., bone mineral density or coronary calcium testing). We also excluded studies that reported only normalized calcium intake, intake in terms of recommended daily allowance (or equivalent), that estimated available dietary calcium (e.g., based on food market surveys), or that reported calcium intake only from selected foodstuffs (e.g., dairy, fruit).

After the full list of potentially relevant studies was collected, we extracted basic study data from the abstracts including 
country, specific location (e.g., if limited to a city), survey name, survey year, sample size, subpopulation data if relevant (e.g., age limitation), and whether subgroup analyses were reported. Based on these data, full-text articles were iteratively retrieved starting with the studies that most closely met ideal eligibility criteria (i.e., that were most representative and recent). Full-text articles were screened to ensure studies met eligibility criteria. Multicountry studies (e.g., EPIC) were evaluated separately for each included country.

\section{Data extraction}

Data were extracted from eligible studies by one researcher and confirmed by a second. We extracted data regarding the publication, country, study location (e.g., city name), survey used and years of survey, methodology for estimation of dietary calcium intake, study eligibility criteria, participant characteristics (age, sex, body mass index), whether children or supplement intake were included, sample size, average estimated dietary calcium intake data, and pertinent subgroup data. Studies were categorized as either nationally representative of current dietary calcium intake (without supplements) among general population adults or not. For countries with multiple estimates of calcium intake (either within study by subgroup, e.g., by sex, or across studies), we determined weighted averages by sample size (or simple averages if sample sizes were not reported) to calculate a single average intake per country. Countries were categorized across the range of average calcium intake. Within-study comparisons of subgroups were evaluated and compared across studies.

\section{Role of the funding source}

The funder of the study had no role in study design, data collection, data analysis, data interpretation, or writing of the report. The corresponding author had full access to all the data in the study and had final responsibility for the decision to submit for publication.

\section{Results}

The literature searches yielded 9780 abstracts after deduplication, of which 443 articles were deemed potentially relevant. Data from two additional nutrition surveys were also included. Our final dataset included 78 eligible studies covering 74 countries (Fig. 1). See Electronic supplementary material-Appendix $\mathrm{C}$ for the full reference list. The most common reasons studies were rejected were a lack of reported calcium data, less restrictive studies available, more recent data available, and other similar reasons. A full list of excluded studies, with rejection reasons, is available in the Electronic supplementary material—Appendix B.
Across the 74 countries with data, the best estimate of average dietary calcium intake among general population adults ranged from $175 \mathrm{mg}$ /day (Nepal) to $1233 \mathrm{mg} /$ day (Iceland) (Table 1). The most notable patterns internationally were that most countries in South, East, and Southeast Asia have low average dietary calcium intake, less than 400 to $500 \mathrm{mg} /$ day (Fig. 2). Most countries with data in Africa and South America have moderately low average dietary calcium intake between about 400 and $700 \mathrm{mg} /$ day. However, many countries in Africa, Central and South America, the Middle East, and Central Asia did not have available estimates. All countries with average dietary calcium intake greater than $1000 \mathrm{mg} /$ day were in Northern Europe.

However, for only 18 of the 74 countries (24\%) were the estimates of dietary calcium intake current (since 2000) and nationally representative (Table 1 ). Thirteen (of 78) study surveys (17\%) were conducted since 2010, 49 studies $(63 \%)$ mostly between 2000 and 2010, 12 studies (15\%) mostly prior to 2000 , and four other studies $(5 \%)$ conducted their surveys at some unreported date prior to about 2004 to 2012. Study sample sizes ranged from 32 to 306,329, with 25 studies (32\%) having fewer than 1000 participants, but 18 studies (23\%) with more than 10,000; three studies did not provide sample sizes. At least 27 of the studies $(35 \%)$ were conducted in specific towns, cities, or regions, but many studies did not report where surveys were conducted. Thirty studies (38\%) included all adults (age 15 years and older), while six studies (8\%) included children, and 39 studies $(50 \%)$ included a limited range of adults. Among 28 studies that reported data on sex, the median study included 59\% women (range 49.6$100 \%$ ), but five were restricted to women. Six studies were conducted at the household (instead of individual) level. Also of note, for two countries with high average calcium intake (Finland and Iceland), the studies explicitly included supplement intake in their estimates; however, for most countries, it was only implied that supplement intake was not included.

Among the studies that reported subgroup analyses based on sex, age, or urban versus rural residence, there were no clear patterns across countries (Table 1). In the 42 countries that had subgroup data by sex, the median calcium intake ratio between women and men was 0.90 (range $0.65,1.18$ ); in 36 $(86 \%)$ countries, women's intake was lower than men's. However, the countries with relatively low calcium intake ratios $(<0.80)$ or with greater intake among women did not fall into any geographic or cultural groupings. In two thirds of countries with reported subgroup estimates by age $(N=21)$, older people consume less dietary calcium, with no discernable patterns across countries. In five of seven mostly lowresource countries, rural residents had lower dietary calcium intake than urban residents. In South Korea, people with worse food sufficiency had lower calcium intake than others, but in Brazil, average dietary calcium intake was similar across income groups. 
Fig. 1 Literature flow chart.

* The 78 studies included two unpublished datasets

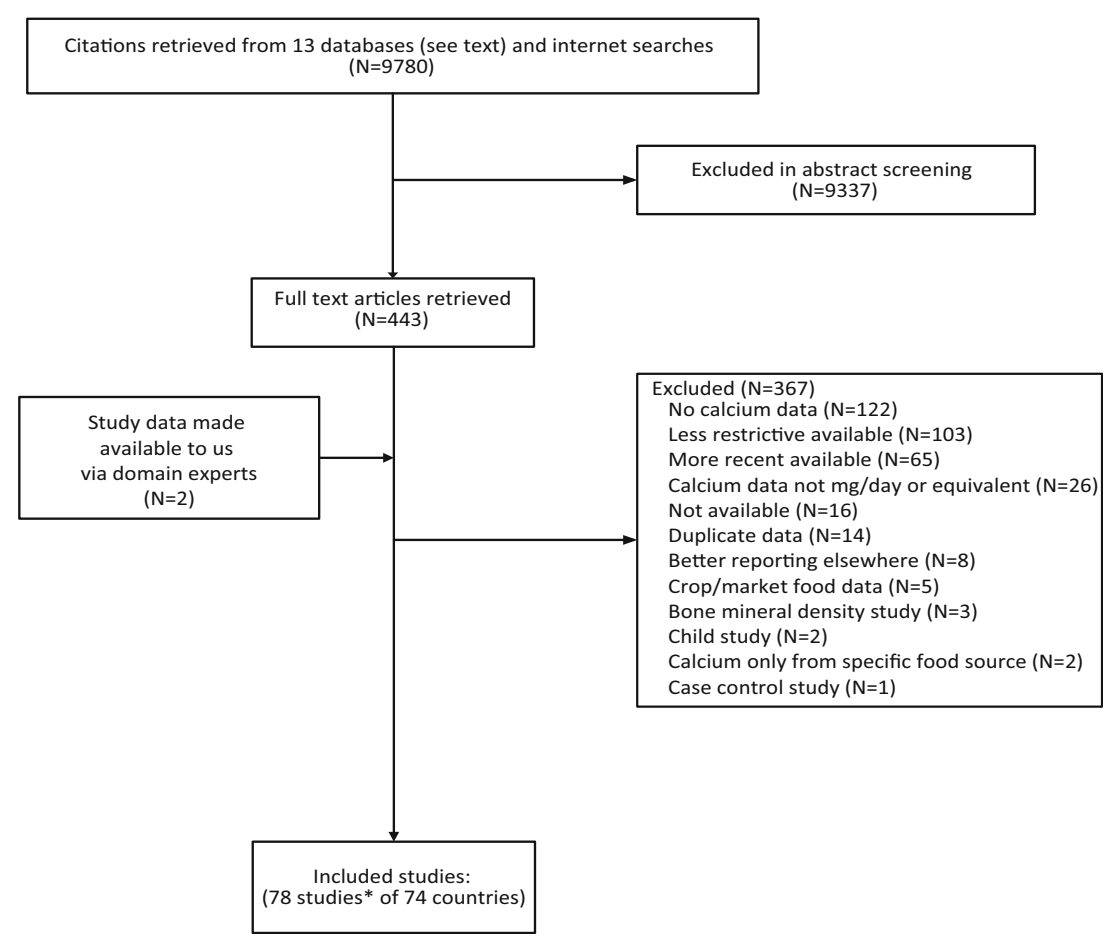

\section{Discussion}

In summary, data on dietary calcium intake were available for 74 countries. However, many of the studies were not nationally representative of general population adults, with about $40 \%$ of countries having only small sample size or local region data available. Most surveyed countries in South, East, and Southeast Asia have low dietary calcium intake (< $400 \mathrm{mg}$ /day). Most surveyed countries in Africa and South America have moderately low calcium intake (400-700 mg/ day). The countries with mean calcium intake greater than $1000 \mathrm{mg}$ /day were all in Northern Europe. Subgroup analyses comparing sexes, ages, and urban versus rural populations did not reveal any consistent patterns across countries.

Review of the global calcium map reveals that there are many countries in which calcium intake is very low, under $400 \mathrm{mg} /$ day. The countries with very low calcium intake are clustered in the Asia-Pacific region and include countries with large populations such as China, India, Indonesia, and Vietnam, among others. Countries in the next lowest intake categories, 400 to 500 and 500 to $600 \mathrm{mg} /$ day, are clustered in South America (Argentina, Bolivia, Brazil) and scattered throughout the Far East, North Africa, and elsewhere. Of the total of 195 countries, we could identify calcium intake data for only 74 or $38 \%$. This leaves 123 countries without qualifying survey data on calcium intake.

Asia-Pacific countries with very low calcium intakes also have suboptimal vitamin D status. In 2012, the IOF published results of a systematic review conducted by the Mannheim Institute describing serum 25-hydroxyvitamin D levels
[25(OH)D] around the globe [3]. Among adults age 18 years and older in most Asia-Pacific countries, mean serum $25(\mathrm{OH}) \mathrm{D}$ levels were in the range of $25-49 \mathrm{nmol} / \mathrm{L}$, which is considered insufficient by the Institute of Medicine, at least for the US general population [6]. The combination of low calcium intake and low $25(\mathrm{OH}) \mathrm{D}$ levels is of particular concern because it is known to increase the risk of osteoporosis. In older adults, for instance, supplementation with calcium in combination with vitamin D reduces bone loss [7], reduces the risk of any fracture [7-9], and specifically reduces the risk of hip fracture $[8,9]$. In much of the Asia-Pacific region where calcium intake is low, serum $25(\mathrm{OH}) \mathrm{D}$ levels also are low [3]. This is notable in China, Malaysia, and India, and also in South Korea where serum $25(\mathrm{OH}) \mathrm{D}$ levels are generally in the range of $25-49 \mathrm{ng} / \mathrm{mL}$ (Table 1).

While the implications of low calcium intake for bone mass have not been systematically evaluated in many countries in the Asia-Pacific region, they have in South Korea. In South Korean adults aged 50 years and older in the 2009-2010 Korean National Health and Examination Survey (KNHANES), Joo et al. examined associations of quintiles of calcium intake with bone mineral density (BMD) within three categories of serum $25(\mathrm{OH}) \mathrm{D}$ levels: $<50,50-75$, and $>$ $75 \mathrm{nmol} / \mathrm{L}$ [10]. Within each category of serum 25(OH)D, higher calcium intake was significantly positively associated with BMD of the femoral neck. Within the lower two $25(\mathrm{OH}) \mathrm{D}$ categories, calcium intake was positively associated with BMD of the spine. These observations in older adults indicate not only that low calcium intake is associated with lower BMD but also that higher $25(\mathrm{OH}) \mathrm{D}$ levels do not 


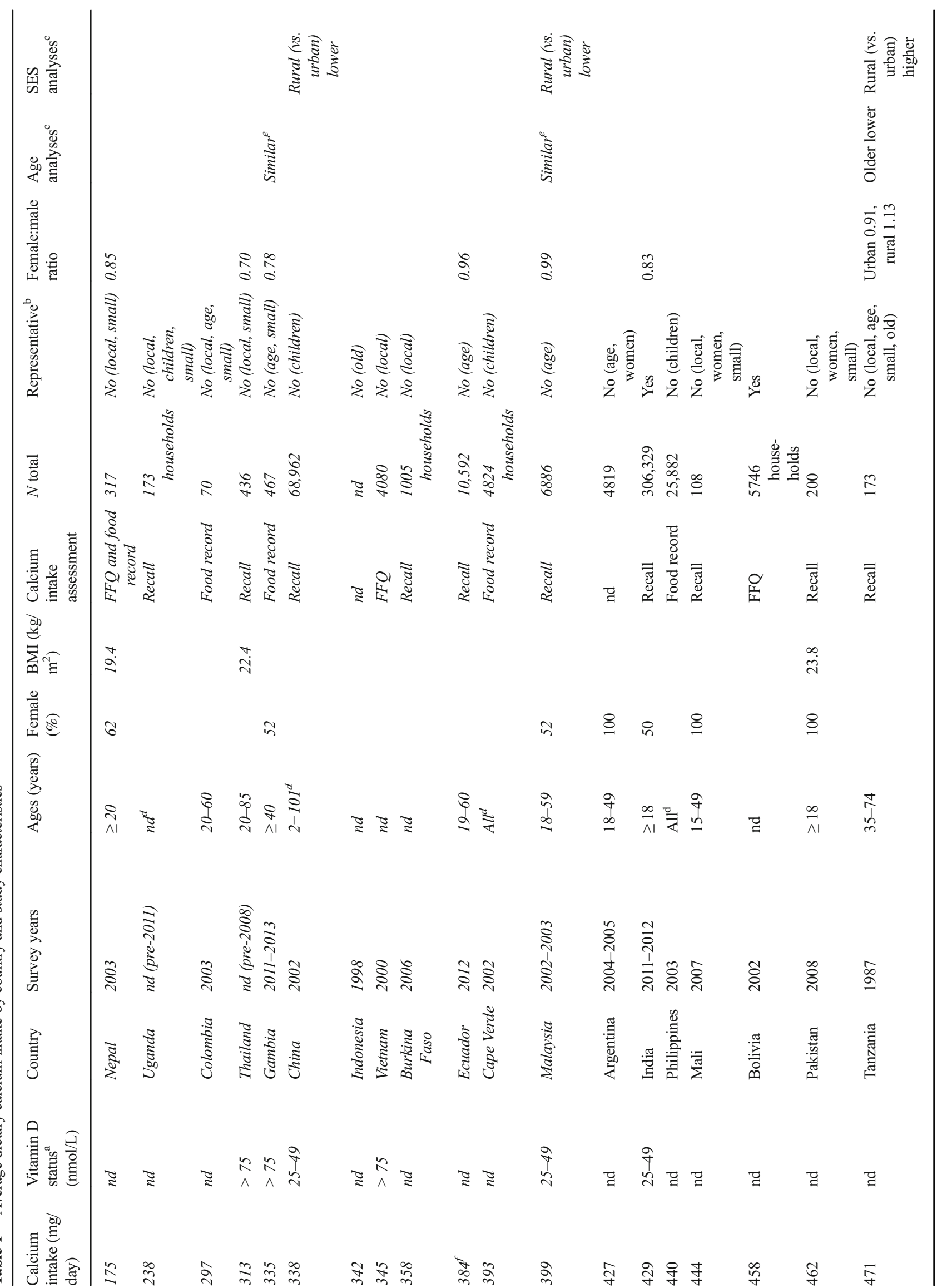




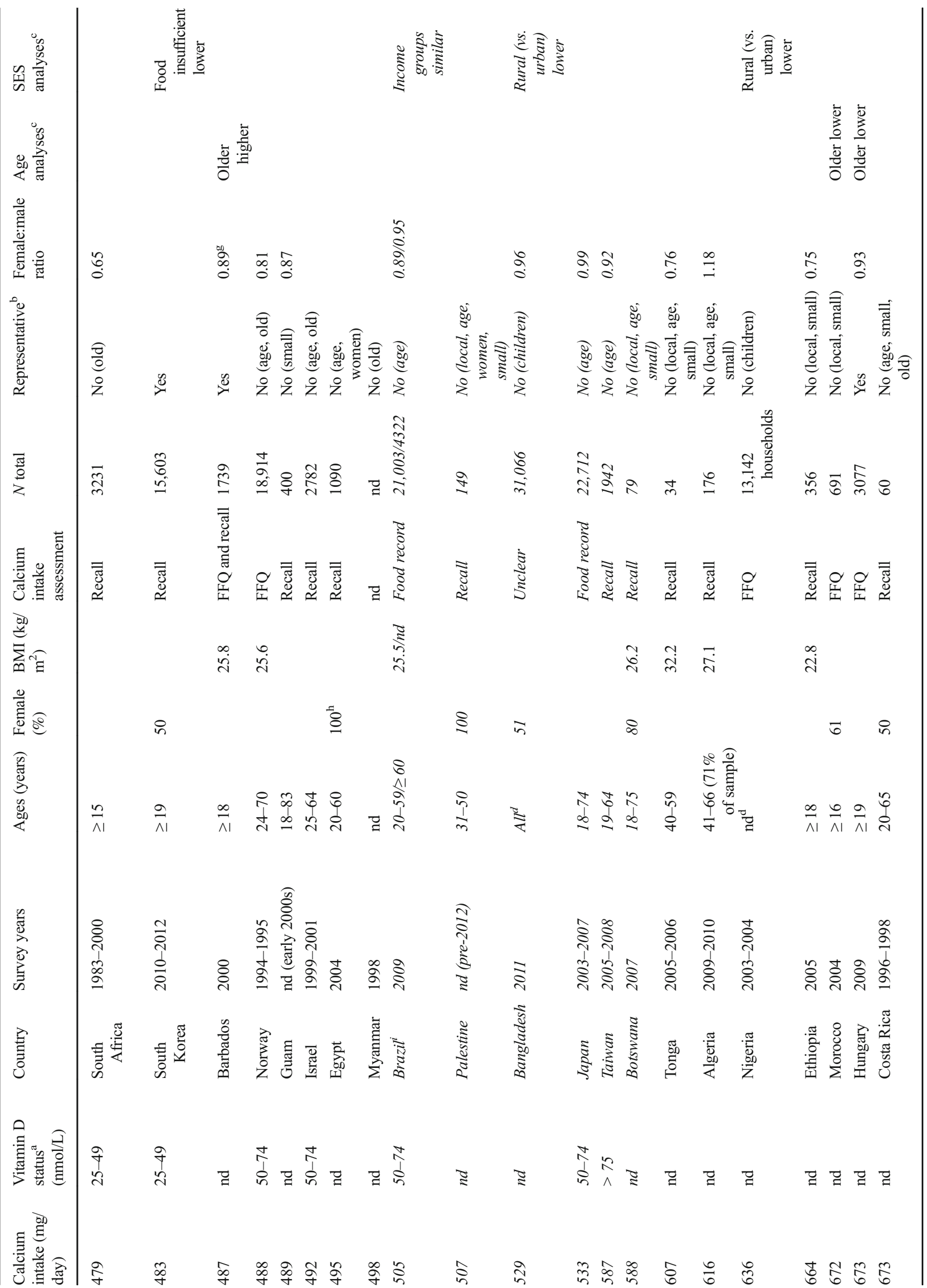




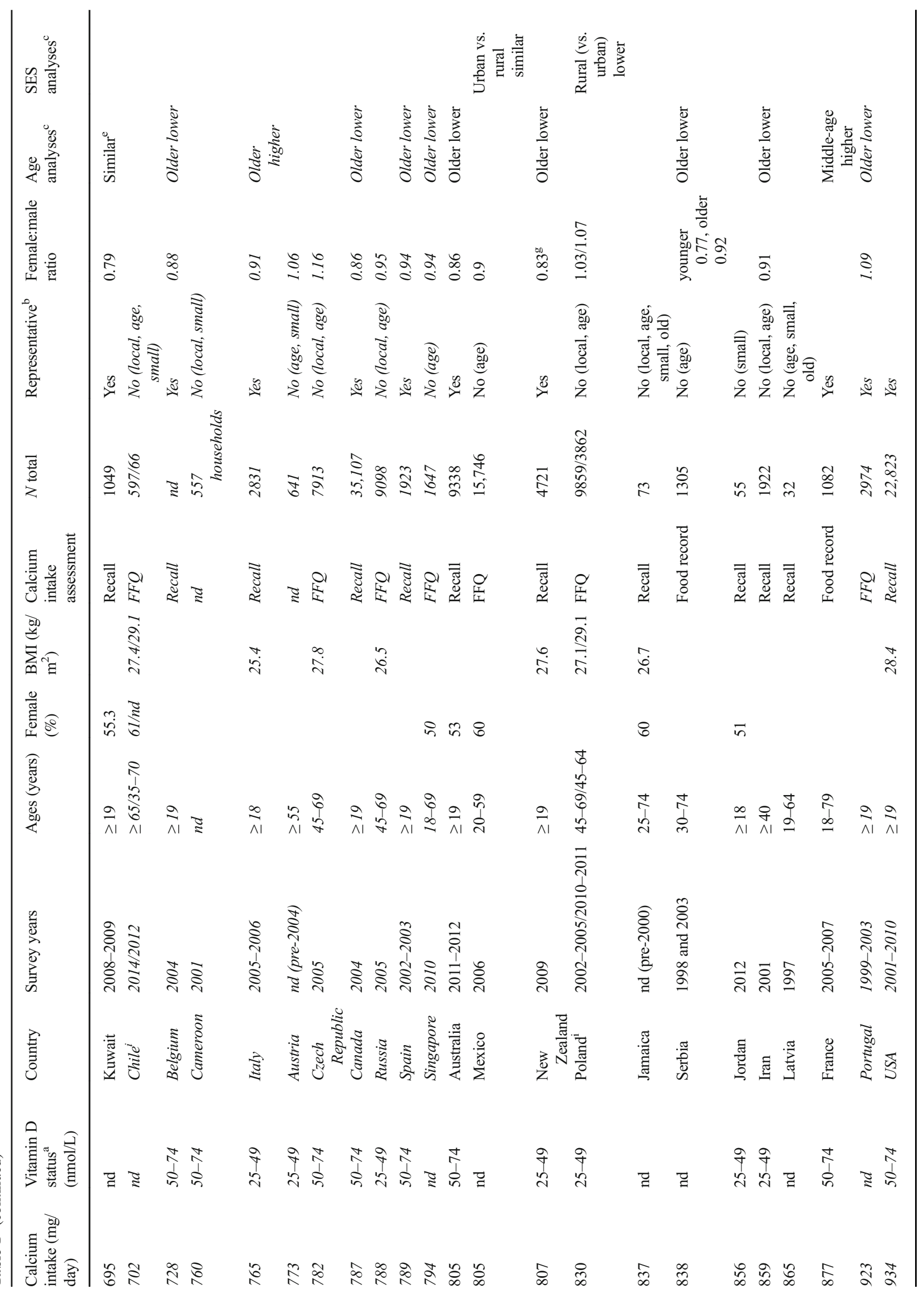




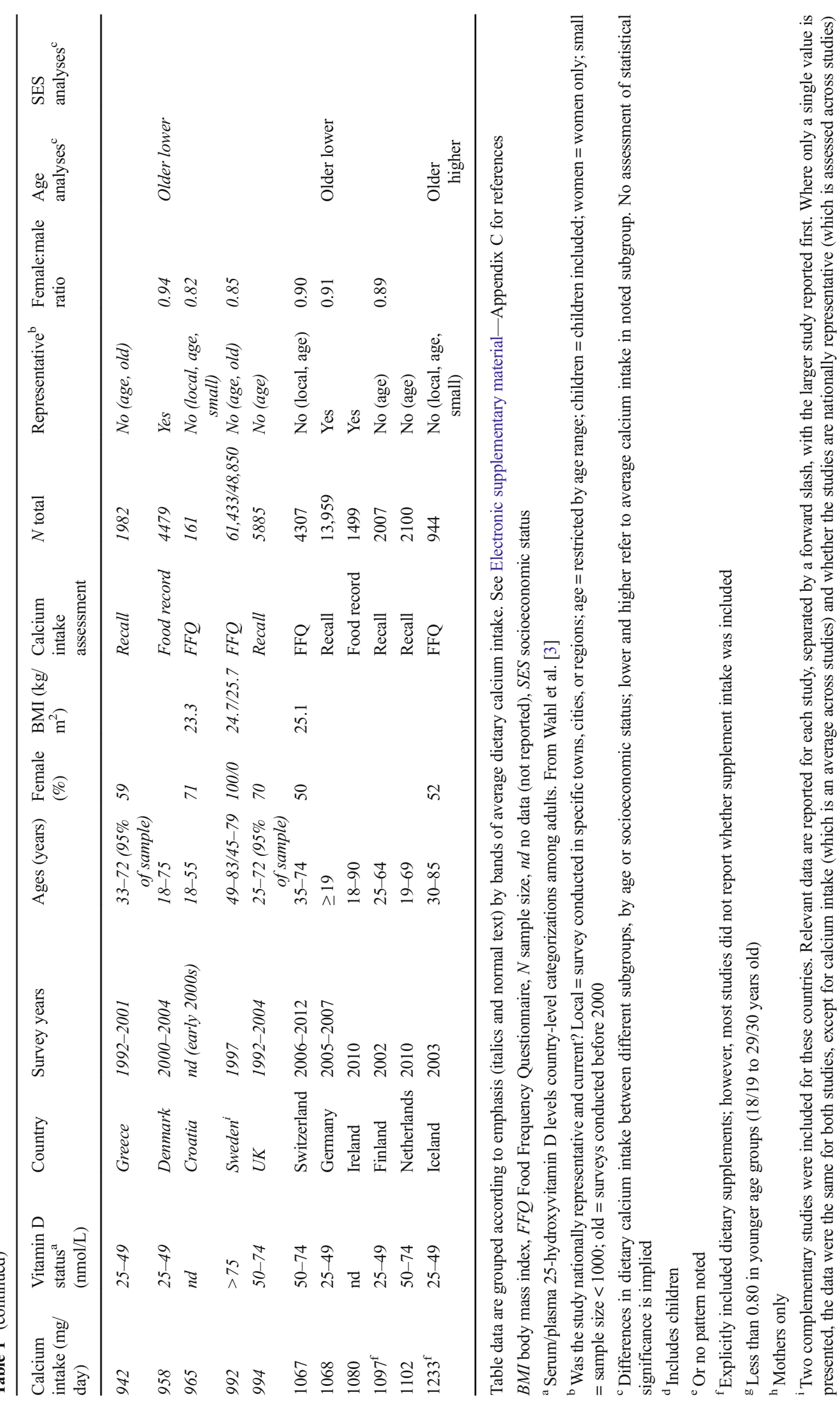




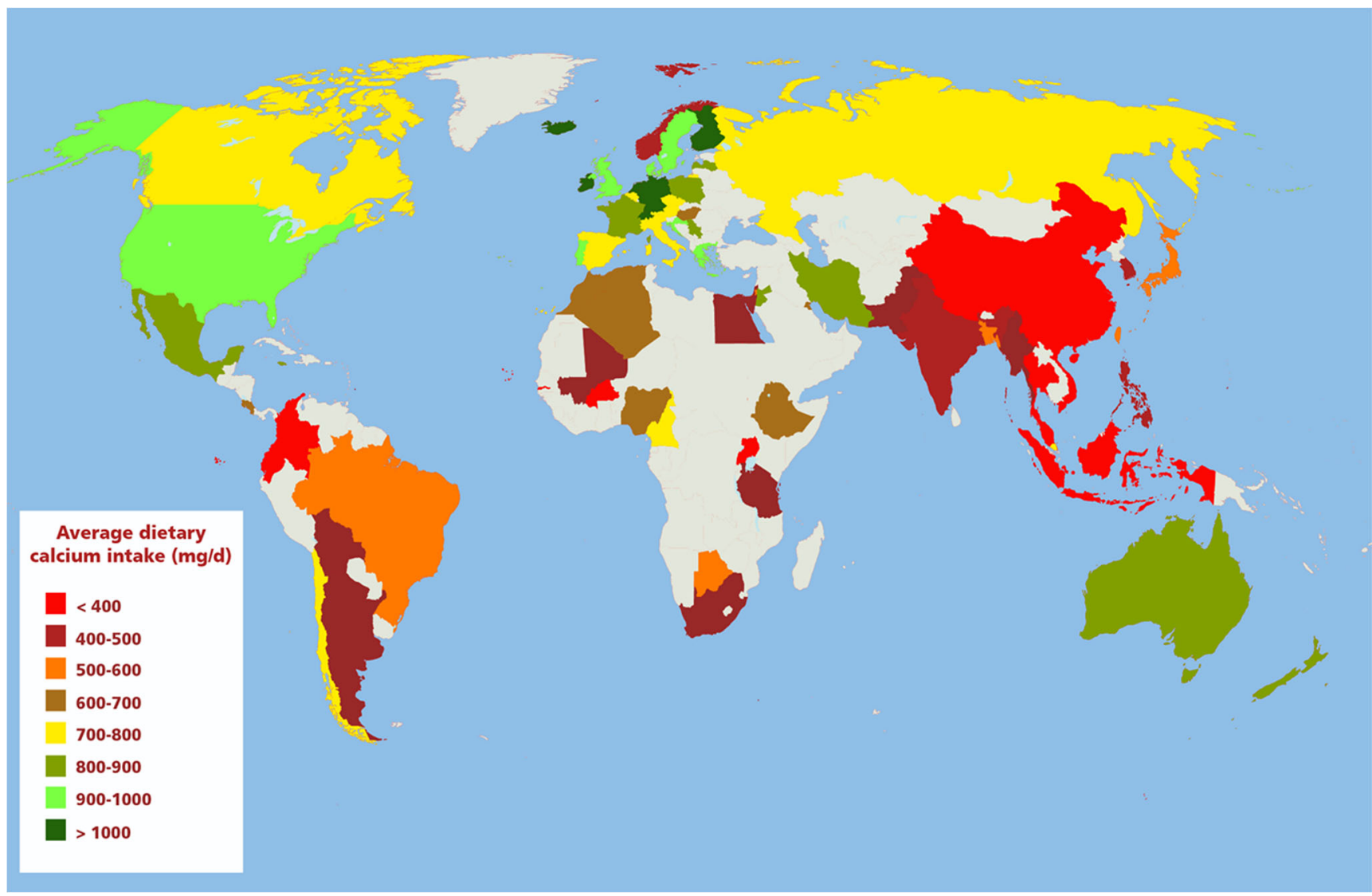

Fig. 2 Global map of average dietary calcium intake categories. Each country with available data is colored based on its estimate of mean or median dietary calcium intake. Bright red $<400 \mathrm{mg}$ /day, dark red 400 $499 \mathrm{mg} /$ day, orange 500-599 mg/day, brown 600-699 mg/day, yellow
700-799 mg/day, moss green 800-899 mg/day, light green 900-999 mg/ day, and dark green $\geq 1000 \mathrm{mg} /$ day. An interactive version of the map is also available online at www.iofbonehealth.org/facts-and-statistics/ calcium-map compensate for an inadequate calcium intake. The mean calcium intake of the older adults was $485 \mathrm{mg} /$ day, and the top quintile consumed at least $666 \mathrm{mg} /$ day. The Korean RDA for calcium for adults age 50 years and older is $700 \mathrm{mg}$ /day [11]. Clearly over $80 \%$ of the older segment of the South Korean population has calcium intake below their RDA. The positive associations of bone mass with calcium intake strongly suggest that a higher calcium intake would improve bone mass in older South Korean adults.

Our goal was to summarize current, representative estimates of dietary calcium intake in adults. However, few countries provided such data. Most studies were not nationally representative or were based on old surveys. Numerous studies had other deficiencies, particularly incomplete reporting of sample size, study eligibility criteria, survey dates, and basic demographic information. Moreover calcium intake was assessed by different methodologies, including food frequency questionnaires, recall, and diet records, which have wellrecognized differences and limitations [12]. For most countries, future, representative, national nutrition surveys are needed to better estimate dietary calcium intake. Notably, we failed to find data for over half the world's nations, particularly from Africa, Asia, and Latin America. Our methodology called for selecting the most representative data for each country; we did not attempt to summarize all available evidence for all countries. Despite clear a priori eligibility criteria, judgments had to be made regarding whether specific studies or articles were less representative than others; for example, whether to choose older, larger studies or newer, smaller studies with a broader eligible age range.

Hip fractures are projected to increase from 1.66 million in 1990 to 6.26 million by 2050 [13]. Europe and North America accounted for about half of all hip fractures in 1990, and this proportion will fall to one quarter in 2050, due to steep increases in reported hip fractures in Asia and South America [13]. Steep increases in Beijing, China, have recently been confirmed [14]. Among adults age 70 years and older, hip fracture rates have increased more than threefold in women 
and twofold in men in the short interval between 1990-1992 and 2002-2006 [14]. Although several dietary, lifestyle, and genetic factors influence hip fracture risk, inadequate calcium intake appears to amplify this risk. To the extent that the current very low calcium intake in Asia-Pacific region and South America adversely affects the skeleton, it becomes a public health priority to increase calcium intake to combat the disabling, growing, and costly problem of osteoporosis in these regions.

In conclusion, this systematic review compiled available data on average national dietary calcium intake around the globe. The key findings are that calcium intake is low (averaging less than $400 \mathrm{mg} /$ day) in many large countries of Southeast Asia and nearly as low in much of South America. Calcium intake has not been reported in over half of the world's countries. This work draws attention to regions where calcium intake needs to be assessed and where measures to increase calcium intake are likely to have skeletal benefits.

Acknowledgements We would like to thank the following researchers for providing or assisting us with data from their studies: Sabri Bromage, MPH (Harvard T.H. Chan School of Public Health, Boston, MA, USA); Ambrish Mithal, MD, DM (Medanta, Gurgaon, Haryana, India); Anura Kurpad, MD, PhD (Division of Nutrition, St. John's Research Institute, Bangalore, India); Peter Ebeling, AO, MBBS, MD, FRACP (Monash University, Clayton, Victoria, Australia); and Kate Ward, PhD (University of Southampton, Southampton, UK).

Funding The study was supported by an unrestricted educational grant to the International Osteoporosis Foundation from Pfizer Consumer Health. The funder had no role in the study design, data collection, analysis, preparation of the manuscript, or decision to publish.

\section{Compliance with ethical standards}

Conflicts of interest Dr. Balk, Ms. Adam, Ms. Langberg, and Ms. Earley report a grant from International Osteoporosis Foundation during the conduct of the study.

Dr. Clark and Dr. Mithal report no conflict of interest.

Dr. Ebeling reports grants and personal fees from Amgen and EliLilly; grants from Novartis and Merck; and personal fees from Gilead, UCB, and Radius, outside the submitted work.

Dr. Rizzoli reports personal fees from Labatec, Nestlé, and Radius Health, outside the submitted work.

Dr. Zerbini reports grants from Pfizer, Lilly, Merck, Amgen, Sanofi Novartis, and Advisory Board work for Lilly and Pfizer, outside the submitted work.

Dr. Pierroz reports grants from Pfizer Consumer Health, during the conduct of the study.

Dr. Dawson-Hughes reports grants from Pfizer and DSM and personal fees from Amgen, Pfizer, Takeda, and Tricida, outside the submitted work.

Open Access This article is distributed under the terms of the Creative Commons Attribution-NonCommercial 4.0 International License (http:// creativecommons.org/licenses/by-nc/4.0/), which permits any noncommercial use, distribution, and reproduction in any medium, provided you give appropriate credit to the original author(s) and the source, provide a link to the Creative Commons license, and indicate if changes were made.

\section{References}

1. Bailey DA, McKay HA, Mirwald RL, Crocker PR, Faulkner RA (1999) A six-year longitudinal study of the relationship of physical activity to bone mineral accrual in growing children: the University of Saskatchewan bone mineral accrual study. J Bone Miner Res 14: $1672-1679$

2. Heaney RP, Recker RR (1982) Effects of nitrogen, phosphorus, and caffeine on calcium balance in women. J Lab Clin Med 99:46-55

3. Wahl DA, Cooper C, Ebeling PR et al (2012) A global representation of vitamin D status in healthy populations. Arch Osteoporos 7: $155-172$

4. Morton S, Berg A, Levit L, Eden J (2011) Finding what works in health care: standards for systematic reviews. National Academies Press, Washington

5. Bromage S, Ahmed T, Fawzi WW (2016) Calcium deficiency in Bangladesh: burden and proposed solutions for the first 1000 days. Food Nutr Bull 37:475-493

6. Institute of Medicine Committee to review dietary reference intakes for vitamin D, calcium (2011) The National Academies Collection: Reports funded by National Institutes of Health. In Ross AC, Taylor CL, Yaktine AL, Del Valle HB (eds) Dietary reference intakes for calcium and vitamin D. National Academies Press (US). National Academy of Sciences, Washington (DC)

7. Tang BM, Eslick GD, Nowson C, Smith C, Bensoussan A (2007) Use of calcium or calcium in combination with vitamin D supplementation to prevent fractures and bone loss in people aged 50 years and older: a meta-analysis. Lancet (London, England) 370: 657-666

8. DIPART (Vitamin D Individual Patient Analysis of Randomized Trials) Group (2010) Patient level pooled analysis of 68500 patients from seven major vitamin D fracture trials in US and Europe. BMJ 340:b5463

9. Weaver CM, Alexander DD, Boushey CJ, Dawson-Hughes B, Lappe JM, LeBoff MS, Liu S, Looker AC, Wallace TC, Wang DD (2016) Calcium plus vitamin D supplementation and risk of fractures: an updated meta-analysis from the National Osteoporosis Foundation. Osteoporos Int 27:367-376

10. Joo NS, Dawson-Hughes B, Kim YS, Oh K, Yeum KJ (2013) Impact of calcium and vitamin D insufficiencies on serum parathyroid hormone and bone mineral density: analysis of the fourth and fifth Korea National Health and Nutrition Examination Survey (KNHANES IV-3, 2009 and KNHANES V-1, 2010). J Bone Miner Res 28:764-770

11. Paik HY (2008) Dietary reference intakes for Koreans (KDRIs). Asia Pac J Clin Nutr 17(Suppl 2):416-419

12. Subar AF, Freedman LS, Tooze JA et al (2015) Addressing current criticism regarding the value of self-report dietary data. J Nutr 145: 2639-2645

13. Cooper C, Campion G, Melton LJ 3rd (1992) Hip fractures in the elderly: a world-wide projection. Osteoporos Int 2:285-289

14. Xia WB, He SL, Xu L, Liu AM, Jiang Y, Li M, Wang O, Xing XP, Sun Y, Cummings SR (2012) Rapidly increasing rates of hip fracture in Beijing, China. J Bone Miner Res 27:125-129 